\title{
PROPER SHAPE INVARIANTS: TAMENESS AND MOVABILITY
}

\author{
ZVONKO ČERIN
}

\author{
University of Zagreb \\ Department of Mathematics \\ Zagreb, 41001 CROATIA \\ zcerin@ 0400 .srce.hr
}

(Received October 8, 1993 and in revised form November 5, 1994).

\begin{abstract}
We study geometric properties of topological spaces called proper $N_{\mathcal{C}}^{\mathcal{B}}$-tameness, proper $P_{\mathcal{C}}^{\mathcal{B}}$-tameness, and proper $N^{\mathcal{B}}$-movability, where $\mathcal{B}$ and $\mathcal{C}$ denote classes of spaces. They are related to proper $M_{\mathcal{C}}^{\mathcal{B}}$-tameness and proper $M^{\mathcal{B}}$-movability from [5] and could be regarded as their dual forms. All three are invariants of a recently invented author's proper shape theory and are described by the use of proper multi-valued functions. We explore their basic properties and prove several results on their preservation under proper maps.
\end{abstract}

KEY WORDS AND PHRASES. Proper multi-valued function, $\sigma$-small, proper $\sigma$-homotopy, proper multi-net, properly $N_{\mathcal{C}}^{\mathcal{B}}$-tame, properly $N_{\mathcal{C}}^{\mathcal{B}}$-tame, properly $N^{\mathcal{B}}$-movable.

1991 AMS SUBJECT CLASSIFICATION. 54B25, 54F45, 54C56.

\section{INTRODUCTION.}

In this paper we shall continue exploration of invariants in the proper shape theory of arbitrary topological spaces from [3]. As in the article [5], we study proper tameness and proper movability. However, this time instead of looking at maps from some objects into a space we take a dual approach where we utilize maps from a space into those objects.

The objects are spaces from classes $\mathcal{B}$ and $\mathcal{C}$ of topological spaces and for maps we take multivalued functions. The dualization procedure when applied to invariants of proper $M_{\mathcal{C}}^{\mathcal{B}}$-tameness and proper $M^{\mathcal{B}}$-movability from [5] gives new invariants which we call proper $N_{\mathcal{C}}^{\mathcal{B}}$-tameness, proper $P_{\mathcal{C}}^{\mathcal{B}}$-tameness and proper $N^{\mathcal{B}}$-movability. The change from the letter $\mathrm{M}$ to the letters $\mathrm{N}$ and $\mathrm{P}$ should reflect duality between these notions. As the reader will see this duality is striking.

Let us describe the content of the paper in greater detail. In $\S 2$ we recall notions and results from Cerin [3] that are necessary in further developments. The next $\S 3$ studies properly $N_{\mathcal{C}}^{\mathcal{B}}$ tame and properly $P_{\mathcal{C}}^{\mathcal{B}}$-tame spaces. The origin for these notions could be traced back to $\mathrm{L}$. Siebenmann's tame at infinity spaces [7]. The idea is that we require that small enough proper multi-valued functions from a given space $X$ into members of a class of spaces $\mathcal{B}$ factor through some member of another class $\mathcal{C}$ also through sufficiently small proper multi-valued functions. This concept is related to the notion of shape dimension Borsuk [2] and could be regarded as a substitute for it in the proper shape theory. We prove that these are invariants in the proper shape category $\mathcal{S} h_{p}$, explore the role of classes $\mathcal{B}$ and $\mathcal{C}$, and study what kind of maps will preserve and inversely preserve properly $N_{\mathcal{C}}^{\mathcal{B}}$-tame and properly $P_{\mathcal{C}}^{\mathcal{B}}$-tame spaces. The classes of proper $M^{\mathcal{B}}$-surjections, proper $N^{\mathcal{B}}$-surjections, proper $M^{\mathcal{B}}$-injections, and proper $N^{\mathcal{B}}$-injections from [4] are of key importance here.

In the following $\S 4$ we consider properly $N^{\mathcal{B}}$-movable spaces which are the analogue of movable compacta Borsuk [2] in proper shape theory.

Finally, in $\S 5$ we consider dependence of these notions on classes $\mathcal{B}$ and $\mathcal{C}$ under the assumption that they are connected with each other by morphisms from [4]. 


\section{PRELIMINARIES ON PROPER SHAPE THEORY.}

In this section we shall introduce notions and results from Cerin [3] that are required for our theory.

Let $X$ and $Y$ be topological spaces. By a multe-valued functeon $F: X \rightarrow Y$ we mean a rule which associates a non-empty subset $F(x)$ of $Y^{-}$to every point $x$ of $X^{\prime}$. A multi-valued function $F: X \rightarrow Y^{\prime}$ is S-proper provided for every compact subset $C$ of $Y$ its small counterimage $F^{\prime}(C)=\left\{x \in X^{\prime} \mid F(x) \subset C^{\prime}\right\}$ is a compact subset of $X^{\prime}$. On the other hand, $F$ is B-proper provided for every compact subset $C$ of $Y^{\prime}$ its big counterimage $F^{\prime \prime}(C)=\{x \in X \mid F(x) \cap C \neq \emptyset\}$ is a compact sulset of $X$. We shall use the term proper to name either $S$-proper or $B$-proper. However, in a given situation, once we make a selection between two different kinds of properness it is understood that it will be retained throughout.

Olsserve that for single-valued functions the two notions of properness coincide. Classes of $S$-proper and $B$-proper multi-valued functions are completely unrelated [3]. It follows that each of our notions and results involving proper multi-valued functions actually has two versions.

Let $\operatorname{Cov}(Y)$ denote the collection of all numerable covers of a topological space $Y$. Let $\operatorname{Inc}(Y)$ denote the collection of all finite subsets $c$ of $\operatorname{Cov}(Y)$ which have a unique (with respect to the refinement relation) maximal element $[c] \in \operatorname{Cov}(Y)$. We consider $\operatorname{Inc}(Y)$ ordered by the inclusion relation and regard $\operatorname{Cov}(Y)$ as a subset of single-element subsets of $\operatorname{Inc}(Y)$.

Let $\sigma \in \operatorname{Cov}(Y)$. Let $\sigma^{+}$denote the set of all numerable covers of $Y$ refining $\sigma$ while $\sigma^{*}$ denotes the set of all numerable covers $\tau$ of $Y$ such that the star st $(\tau)$ of $\tau$ refines $\sigma$. Similarly, for a natural number $n, \sigma^{* n}$ denotes the set of all numerable covers $\tau$ of $Y$ such that the $n$-th star $s t^{n}(\tau)$ of $\tau$ refines $\sigma$.

For our approach to proper shape theory the following notion of size for multi-valued functions will play the most important role.

Let $F: X \rightarrow Y$ be a multi-valued function and let $\alpha \in \operatorname{Cov}(X)$ and $\gamma \in \operatorname{Cov}(Y)$. We shall say that $F$ is an $(\alpha, \gamma)$-function provided for every $A \in \alpha$ there is a $C_{A} \in \gamma$ with $F(A) \subset C_{A}$. On the other hand, $F$ is $\gamma$-small provided there is an $\alpha \in \operatorname{Cov}(X)$ such that $F$ is an $(\alpha, \gamma)$ function. For a $\sigma$-small multi-valued function $F: X \rightarrow Y$ we use $S(F, \sigma)$ to denote the family of all numerable covers $\alpha$ of $X$ such that $F$ is an $(\alpha, \sigma)$-function.

Next we introduce the notions which correspond to the equivalence relation of proper homotopy for proper maps.

Let $F$ and $G$ be proper multi-valued functions from a space $X$ into a space $Y$ and let $\gamma$ be a numerable cover of $Y$. We shall say that $F$ and $G$ are properly $\gamma$-homotopic and write $F \stackrel{\gamma}{=} G$ provided there is a $\gamma$-small proper multi-valued function $H$ from the product $X \times I$ of $X$ and the unit segment $I=[0,1]$ into $Y$ such that $F(x) \subset H(x, 0)$ and $G(x) \subset H(x, 1)$ for every $x \in X$. We shall say that $H$ is a proper $\gamma$-homotopy that joins $F$ and $G$ or that it realizes the relation (or proper homotopy) $F \stackrel{\gamma}{=} G$.

The following two definitions correspond to Ball and Sher's definitions of proper fundamental net and proper homotopy for proper fundamental nets from [1].

Let $X$ and $Y$ be topological spaces. By a proper multi-net from $X$ into $Y$ we shall mean a collection $\varphi=\left\{F_{c} \mid c \in \operatorname{Inc}(Y)\right\}$ of proper multi-valued functions $F_{c}: X \rightarrow Y$ such that for every $\gamma \in \operatorname{Cov}(Y)$ there is a $c \in \operatorname{Inc}(Y)$ with $F_{d} \stackrel{\gamma}{=} F_{c}$ for every $d>c$. We use functional notation $\varphi: X \rightarrow Y$ to indicate that $\varphi$ is a proper multi-net from $X$ into $Y$. Let $M_{p}(X, Y)$ denote all proper multi-nets $\varphi: X \rightarrow Y$.

Two proper multi-nets $\varphi=\left\{F_{c}\right\}$ and $\psi=\left\{G_{c}\right\}$ between topological spaces $X$ and $Y$ are properly homotopic and we write $\varphi \bumpeq \psi$ provided for every $\gamma \in \operatorname{Cov}(Y)$ there is a $c \in \operatorname{Inc}(Y)$ such that $F_{d} \stackrel{\gamma}{=} G_{d}$ for every $d>c$. On the other hand, we write $\varphi \stackrel{\gamma}{\bumpeq} \psi$, and call $\varphi$ and $\psi$ properly $\gamma$-homotopic provided there is a $c \in \operatorname{Inc}(Y)$ such that $F_{d} \stackrel{\gamma}{=} G_{d}$ for every $d>c$.

The relation of proper homotopy is an equivalence relation on the set $M_{p}(X, Y)$. The proper homotopy class of a proper multi-net $\varphi$ is denoted by $[\varphi]$ and the set of all proper homotopy classes by $\mathcal{S} h_{p}(X, Y)$.

The topological spaces as objects together with the proper homotopy classes of proper multi-nets as morphisms form the proper shape category $\mathcal{S} h_{p}$ (see [3]).

\section{PROPERLY $N_{\mathcal{C}}^{\mathcal{B}}$-TAME AND PROPERLY $P_{\mathcal{C}}^{\mathcal{B}}$-TAME CLASSES.}

The notion of a properly $M_{\mathcal{C}}^{\mathcal{B}}$-tame class of spaces from [5] allow us to obtain two new properties that are preserved under proper $M$-domination. They could be considered as dual 
to the notion of a properly $M_{C^{-}}^{\mathcal{B}}$-tame space. While in [5] we investigated a space $X$ by looking at small proper multi-valued functions from members of a given class of spaces $\mathcal{B}$ into $X$, we now change our point of view by concentrating on small proper multi-valued functions from $X$ into members of $\mathcal{B}$.

Let $\mathcal{B}$ and $\mathcal{C}$ be classes of spaces. A class of spaces $\mathcal{X}$ is (1) properly $N_{\mathcal{C}}^{\mathcal{B}}$-tame and (2)

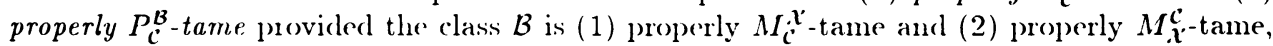
respectively. In other words, provided that

(1) for every $B \in \mathcal{B}$ and every $\sigma \in \operatorname{Cov}(B)$ there is a $\tau \in \operatorname{Cov}(B)$ such that for every $X \in \mathcal{X}$ and every proper $\tau$-small multi-valued function $F: X \rightarrow B$ there is a $C \in \mathcal{C}$ and a proper $\sigma$-small multi-valued function $H: C \rightarrow B$ so that for every $\gamma \in \operatorname{Cov}(C)$ there is a proper $\gamma$-small multi-valued function $G: X \rightarrow C$ with $F \stackrel{\sigma}{=} H \circ G$.

(2) for every $B \in \mathcal{B}$ and every $\sigma \in \operatorname{Cov}(B)$ there is a $\tau \in \operatorname{Cov}(B)$ such that for every $C \in \mathcal{C}$ and every proper $\tau$-small multi-valued function $F: C \rightarrow B$ there is an $X \in \mathcal{X}$ and a proper $\sigma$-small multi-valued function $H: X \rightarrow B$ so that for every $\xi \in \operatorname{Cov}(X)$ there is a proper $\xi$-small multi-valued function $G: C \rightarrow X$ with $F \stackrel{\sigma}{=} H \circ G$.

We shall say that a space $X$ has one of the above properties provided the class $\{X\}$ consisting just of a space $X$ has this property.

The three versions of proper tameness share many properties. We shall now state and prove the $\mathrm{N}$ and the $\mathrm{P}$ versions of most results from $\S 3$ in [5].

THEOREM 3.1. A class $\mathcal{X}$ of spaces is properly $N_{\mathfrak{C}}^{\mathcal{B}}$-tame if and only if a properly $N_{\mathfrak{C}^{-}}^{\mathcal{B}}$ tame class of spaces properly $M$-dominates it.

PROOF. Suppose that $\mathcal{X}$ is properly $M$-dominated by a properly $N_{\mathcal{C}}^{\mathcal{B}}$-tame class $\mathcal{Y}$. Then $\mathcal{B}$ is properly $M_{\mathcal{C}}^{\mathcal{Y}}$-tame so that $\mathcal{B}$ is properly $M_{\mathcal{C}}^{\mathcal{X}}$-tame by theorem 3.5 in [5]. Hence, $\mathcal{X}$ is properly $N_{\mathcal{C}}^{\mathcal{B}}$-tame.

COROLLARY 3.2. A space is properly $N_{\mathcal{C}}^{\mathcal{B}}$-tame if and only if it is quasi $\mathcal{S} h_{p}$-dominated or $S h_{p}$-dominated by a properly $N_{\mathcal{C}}^{\mathcal{B}}$-tame class of spaces.

THEOREM 3.3. A class $\mathcal{X}$ of spaces is properly $P_{\mathcal{C}}^{\mathcal{B}}$-tame if and only if a properly $P_{\mathcal{C}}^{\mathcal{B}}$-tame class of spaces properly $M$-dominates it.

PROOF. Similar to the proof of theorem 3.1.

COROLLARY 3.4. A space is properly $P_{\mathcal{C}}^{\mathcal{B}}$-tame if and only if it $S h_{p}$-dominates or quasi $S h_{p}$-dominates a properly $P_{\mathcal{C}}^{\mathcal{B}}$-tame class of spaces.

THEOREM 3.5. Let $\mathcal{A}, \mathcal{B}$, and $\mathcal{C}$ be classes of spaces. If a class $\mathcal{X}$ of spaces is both properly $N_{\mathcal{A}}^{\mathcal{B}}$-tame and properly $N_{\mathcal{C}}^{\mathcal{A}}$-tame, then $\mathcal{X}$ is also properly $N_{\mathcal{C}}^{\mathcal{B}}$-tame.

PROOF. Let a member $B$ of $\mathcal{B}$ and a numerable cover $\sigma$ of $B$ be given. Let $\mu \in \sigma^{*}$. Since $X$ is properly $N_{\mathcal{A}}^{\mathcal{B}}$-tame, there is a $\tau \in \operatorname{Cov}(B)$ such that for every $X \in \mathcal{X}^{\prime}$ and every proper $\tau$-small multi-valued function $F: X \rightarrow B$ there is a $A \in \mathcal{A}$ and a proper $\mu$-small multi-valued function $K: A \rightarrow B$ so that for every $\xi \in \operatorname{Cov}(A)$ there is a proper $\gamma$-small multi-valued function $L: X \rightarrow A$ with $F \stackrel{\mu}{=} L \circ K$. Then $\tau$ is the cover we have been looking for.

Indeed, let $X \in \mathcal{X}$ and let $F: X \rightarrow B$ be a proper $\tau$-small multi-valued function. Pick an $A \in \mathcal{A}$ and a $K$ as above. Let $\theta \in S(K, \mu)$. Now we utilize the fact that $\mathcal{X}$ is also properly $N_{\mathcal{C}}^{\mathcal{A}}$-tame to select a numerable cover $\xi$ of $A$ such that for every $X \in \mathcal{X}$ and every proper $\xi$-small multi-valued function $L: X \rightarrow A$ there is a $C \in \mathcal{C}$ and a proper $\theta$-small multi-valued function $M: C \rightarrow A$ so that for every $\gamma \in \operatorname{Cov}(C)$ there is a proper $\gamma$-small multi-valued function $G: X \rightarrow C$ with $L \stackrel{\theta}{=} M \circ G$. Choose an $L$ and then a $C$ and an $M$ as above. Let $H$ denotes the composition $K \circ M$. Then $C$ and $H$ are the required space and proper function.

In fact, let a $\gamma \in \mathcal{C}$ be given. We first choose a $G$ as above. Our choices give us the following chain of relations $F \stackrel{\mu}{=} L \circ K \stackrel{\mu}{\bumpeq} K \circ M \circ G=H \circ G$. Hence, $F \stackrel{\sigma}{=} H \circ G$.

THEOREM 3.6. Let $\mathcal{A}, \mathcal{B}$, and $\mathcal{C}$ be classes of spaces. If a class $\mathcal{X}$ of spaces is properly $P_{\mathcal{A}}^{\mathcal{B}}$-tame and the class $\mathcal{B}$ is properly $M_{\mathcal{A}}^{\mathfrak{c}^{\prime}}$-tame, then $\mathcal{X}^{\prime}$ is also properly $P_{\mathcal{C}}^{\mathcal{B}}$-tame.

PROOF. The assumption on $\mathcal{X}$ can be expressed by saying that the class $\mathcal{B}$ is properly $M_{\mathcal{X}}^{\mathcal{A}}$-tame. It follows from theorem 3.4 in [5] that $\mathcal{B}$ is properly $M_{\mathcal{X}^{\mathcal{C}}}^{\mathcal{C}}$-tame or that $\mathcal{X}^{\prime}$ is properly $P_{\mathcal{C}}^{\mathcal{B}}$-tame. 
THEOREM 3.T. Let $\mathcal{A}, \mathcal{B}, \mathcal{C}$, and $\mathcal{D}$ be classes of spaces such that $\mathcal{B}$ and $\mathcal{D}$ are properly $M$-dominated by $\mathcal{A}$ and $\mathcal{C}$, respectively. If a class . $\mathcal{X}^{\prime}$ of spaces is properly $N_{\mathcal{D}}^{\mathcal{A}}$-tame, then it is also properly $N_{\mathcal{C}}^{B}$-tame.

PROOF. The assumption that $\chi \chi^{\prime}$ is properly $M_{\mathcal{D}}^{\mathcal{A}}$-tame means that $\mathcal{A}$ is properly $M_{\mathcal{D}^{-}}^{\mathcal{X}^{\prime}}$ tame. Since $\mathcal{A}$ properly $M$-dominates $\mathcal{B}$, it follows from theorem 3.1 in [5] that $\mathcal{B}$ is properly $M_{\mathcal{D}}^{\mathcal{X}^{\prime}}$-tame. But, since $\mathcal{C}$ properly $M$-dominates $\mathcal{D}$, we get that $\mathcal{B}$ is properly $M_{\mathcal{C}}^{\mathcal{X}^{\prime}}$-tame and therefore that $\mathcal{X}^{\prime}$ is properly $N_{\mathfrak{C}}^{\mathcal{B}}$-tame.

COROLLARY 3.8. Let $\mathcal{A}, \mathcal{B}, \mathcal{C}$, and $\mathcal{D}$ be classes of spaces such that $\mathcal{B}$ and $\mathcal{D}$ are quasi $S h_{p}$-dominated or $\mathcal{S} h_{p}$-dominated by $\mathcal{A}$ and $\mathcal{C}$, respectively. If a space $X$ is properly $N_{\mathcal{D}}^{\mathcal{A}}$-tame, then it is also properly $N_{\mathcal{C}}^{\mathcal{B}}$-tame.

THEOREM 3.9. Let $\mathcal{A}, \mathcal{B}, \mathcal{C}$, and $\mathcal{D}$ be classes of spaces such that $\mathcal{B}$ and $\mathcal{C}$ are properly $M$-dominated by $\mathcal{A}$ and $\mathcal{D}$, respectively. If a class $\mathcal{X}$ of spaces is properly $P_{\mathcal{D}}^{\mathcal{A}}$-tame, then it is also properly $P_{\mathcal{C}}^{\mathcal{B}}$-tame.

PROOF. See the proof of theorem 3.7.

COROLLARY 3.10. Let $\mathcal{A}, \mathcal{B}, \mathcal{C}$, and $\mathcal{D}$ be classes of spaces such that $\mathcal{B}$ and $\mathcal{C}$ are quasi $\mathcal{S} h_{p}$-dominated or $\mathcal{S} h_{p}$-dominated by $\mathcal{A}$ and $\mathcal{D}$, respectively. If a space $X$ is properly $P_{\mathcal{D}}^{\mathcal{A}}$-tame, then it is also properly $P_{\mathcal{C}}^{\mathcal{B}}$-tame.

There seems to be no analogue of theorem 3.7 in [5] for properly $N_{\mathcal{C}}^{\mathcal{B}}$-tame and properly $P_{\mathcal{C}}^{\mathcal{B}}$-tame spaces. In order to state versions of theorem 3.9 in [5] we need the following dual form of the notion of a proper $M^{\mathcal{B}}$-surjection from [4].

Let $\mathcal{B}$ be a class of topological spaces. A class $\mathcal{F}$ of proper maps is properly $N^{\mathcal{B}}$-surjective provided for every $B \in \mathcal{B}$ and every $\sigma \in \operatorname{Cov}(B)$ there is a $\tau \in \operatorname{Cov}(B)$ such that for every $f: X \rightarrow Y$ from $\mathcal{F}$ and every proper $\tau$-small multi-valued function $F: X \rightarrow B$ there is a proper $\sigma$-small multi-valued function $G: Y \rightarrow B$ with $F \stackrel{\sigma}{\simeq} G \circ f$. A proper map $f: X \rightarrow Y$ is a proper $N^{\mathcal{B}}$-surjection provided the class $\{f\}$ is properly $N^{\mathcal{B}}$-surjective.

THEOREM 3.11. If $\mathcal{F}$ is a properly $N^{\mathcal{B}}$-surjective class of proper maps and the class $\mathcal{F}^{\prime \prime}$ is properly $N_{\mathcal{C}}^{\mathcal{B}}$-tame, then the class $\mathcal{F}^{\prime}$ is also a properly $N_{\mathcal{C}}^{\mathcal{B}}$-tame.

PROOF. Let a member $B$ of $\mathcal{B}$ and a numerable cover $\sigma$ of $B$ be given. Let $\mu \in \sigma^{*}$. Since $\mathcal{F}^{\prime \prime}$ is properly $N_{\mathcal{C}}^{\mathcal{B}}$-tame, there is a $\xi \in \mu^{+}$such that for every $Y \in \mathcal{F}^{\prime \prime}$ and every proper $\xi$-small multi-valued function $K: Y \rightarrow B$ there is a $C \in \mathcal{C}$ and a proper $\mu$-small multi-valued function $H: C \rightarrow B$ so that for every $\gamma \in \operatorname{Cov}(C)$ there is a proper $\gamma$-small multi-valued function $L: Y \rightarrow C$ so that $K \stackrel{\mu}{\bumpeq} H \circ L$. Finally, we utilize the fact that $\mathcal{F}$ is a properly $N^{\mathcal{B}}$-surjective to select the required numerable cover $\tau$ of $B$ so that for every member $f: X \rightarrow Y$ of $\mathcal{F}$ and every proper $\tau$-small multi-valued function $F: X \rightarrow B$ there is a proper $\xi$-small multi-valued function $K: Y \rightarrow B$ with $F \stackrel{\xi}{=} K \circ f$.

Consider a proper $\tau$-small multi-valued function $F: X \rightarrow B$ from a member $X$ of $\mathcal{F}^{\prime}$ into $B$. Choose a proper map $f: X \rightarrow Y$ from $\mathcal{F}$, a $K$, and then a $C$ and an $H$ as above. Let $\gamma \in \operatorname{Cov}(C)$. Pick an $L$ and let $G$ denote the composition of $f$ and $L$. Notice that $G$ is a proper $\gamma$-small multi-valued function. Then we obtain the following chain of relations. $F \stackrel{\xi}{=} K \circ f \stackrel{\mu}{=} H \circ L \circ f=H \circ G$. Hence, $F \stackrel{\sigma}{=} H \circ G$ and $\mathcal{F}^{\prime}$ is properly $\mathcal{B C} N$-tame.

In a similar fashion one can prove the following result for properly $P_{\mathcal{C}}^{\mathcal{B}}$-tame spaces.

THEOREM 3.12. If $\mathcal{F}$ is a properly $N^{\mathcal{B}}$-surjective class of proper maps and the class $\mathcal{F}^{\prime}$ is properly $P_{\mathcal{C}}^{\mathcal{B}}$-tame, then the class $\mathcal{F}^{\prime \prime}$ is also a properly $P_{\mathcal{C}}^{\mathcal{B}}$-tame.

THEOREM. 3.13. If $\mathcal{F}$ is a class of properly right $M$-placid proper maps and the class $\mathcal{F}^{\prime}$ is properly $N_{\mathcal{C}}^{\mathcal{B}}$-tame, then the class $\mathcal{F}^{\prime \prime}$ is also properly $N_{\mathcal{C}}^{\mathcal{B}}$-tame.

PROOF. Let a member $B$ of $\mathcal{B}$ and a numerable cover $\sigma$ of $B$ be given. Let $\mu \in \sigma^{*}$. Since $\mathcal{F}^{\prime}$ is properly $N_{\mathcal{C}}^{\mathcal{B}}$-tame, there is a $\tau \in \mu^{+}$such that for every $X \in \mathcal{F}^{\prime}$ and every proper $\tau$-small multi-valued function $P: X \rightarrow B$ there is a $C \in \mathcal{C}$ and a proper $\mu$-small multi-valued function $H: C \rightarrow B$ so that for every $\gamma \in \operatorname{Cov}(C)$ there is a proper $\gamma$-small multi-valued function 
$Q: \mathrm{X} \rightarrow C$ and a proper $\mu$-homotopy $W$ joining $P$ and $H \circ Q$. Then $\tau$ is the required cover. Indeed, consider a member $Y^{-}$of $\mathcal{F}^{\prime \prime}$ and a proper $\tau$-small multi-valued function $F: Y \rightarrow B$. Let $f: X \rightarrow Y$ be from the class $\mathcal{F}$. Let $P$ denote the composition of $f$ and $F$. Then $P$ is a proper $\tau$-small multi-valued function from $X$ into $B$ so that a $C \in \mathcal{C}$ and an $H$ as above exist.

Let a $\gamma \in \operatorname{Cov}(C)$ be given. Now, we pick the function $Q$ and a proper $\mu$-homotopy $W$. Let $\lambda \in S(F, \tau)$ and let $\theta$ be from the intersection of sets $D(W, \mu)$ and $S(Q, \gamma)$. Since $f$ is properly right $M$-placid, there is a proper $\theta$-small multi-valued function $J: Y \rightarrow X$ such that $f \circ J \stackrel{\lambda}{\bumpeq} \imath d_{Y}$. Let $G$ denote the composition of $J$ and $Q$. Then we have the following relations. $F \stackrel{\tau}{=} F \circ f \circ J \stackrel{\mu}{=} H \circ Q \circ J=G \circ H$. Hence, $F \stackrel{\sigma}{=} H \circ G$.

COROLLARY 3.14. A proper $M$-retract of a properly $N_{\mathfrak{C}}^{\mathcal{B}}$-tame space is properly $N_{\mathfrak{c}}^{\mathcal{B}}$ tame.

THEOREM 3.15. If $\mathcal{F}$ is a class of properly right $M$-placid proper maps and the class $\mathcal{F}^{\prime \prime}$ is properly $N_{\mathcal{C}}^{\mathcal{B}}$-tame, then the class $\mathcal{F}^{\prime}$ is also properly $N_{\mathcal{C}}^{\mathcal{B}}$-tame.

PROOF. The proof is similar to the proof of theorem 3.13.

In the next result that corresponds to theorem 3.13 in [5] we shall use a notion of properly $N^{\mathcal{B}}$-injective class of proper maps from [4] whose definition we now recall.

Let $\mathcal{B}$ be a class of spaces. A class $\mathcal{F}$ of proper maps is properly $N^{\mathcal{B}}$-injective provided for every $B \in \mathcal{B}$ and every $\sigma \in \operatorname{Cov}(B)$ there is a $\tau \in \operatorname{Cov}(B)$ such that for every $f: X \rightarrow Y$ from $\mathcal{F}$ and for every proper $\tau$-small multi-valued functions $F, G: Y \rightarrow B$ the relation $F \circ f \stackrel{\tau}{\simeq} G \circ f$ implies the relation $F \stackrel{\sigma}{=} G$. A proper map $f: X \rightarrow Y$ is a proper $N^{\mathcal{B}}$-injection provided the class $\{f\}$ consisting of $f$ alone is properly $N^{\mathcal{B}}$-injective.

Also, a class of proper maps which is both properly $N^{\mathcal{B}}$-injective and properly $N^{\mathfrak{c}}$-surjective is called properly $N_{\mathfrak{c}}^{\mathcal{B}}$-bijective. We shall use a shorter name properly $N^{\mathcal{B}}$-bijective for a properly $N_{\mathcal{B}}^{\mathcal{B}}$-bijective class of proper maps. A proper map $f$ is a proper $N_{\mathcal{C}}^{\mathcal{B}}$-bijection provided the class $\{f\}$ is properly $N_{c^{\mathcal{B}}}^{\mathcal{B}}$-bijective. A proper $N^{\mathcal{B}}$-bijectıon is defined analogously.

THEOREM 3.16. If $\mathcal{F}$ is a properly $N^{\mathcal{B}}$-injective class of proper $N^{\mathcal{C}}$-surjections and the class $\mathcal{F}^{\prime}$ is properly $N_{\mathcal{C}}^{\mathcal{B}}$-tame, then the class $\mathcal{F}^{\prime \prime}$ is also properly $N_{\mathfrak{C}}^{\mathcal{B}}$-tame.

PROOF. Let a member $B$ of $\mathcal{B}$ and a numerable cover $\sigma$ of $B$ be given. Since $\mathcal{F}$ is properly $N^{\mathcal{B}}$-injective, there is a $\varrho \in \operatorname{Cov}(B)$ such that for every map $f: X \rightarrow Y$ from the class $\mathcal{F}$ and all proper $\varrho$-small multi-valued functions $P, Q: Y \rightarrow B$ the relation $P \circ f \stackrel{\varrho}{=} Q \circ f$ implies the relation $P \stackrel{\sigma}{=} Q$. Let $\mu \in \varrho^{*}$. We now utilize the assumption that $\mathcal{F}^{\prime}$ is properly $N_{\mathcal{C}}^{\mathcal{B}}$-tame to select a $\tau \in \operatorname{Cov}(B)$ such that for every member $X$ of the class $\mathcal{F}^{\prime}$ and every proper $\tau$-small multi-valued function $K: X \rightarrow B$ there is a $C \in \mathcal{C}$ and a proper $\mu$-small multi-valued function $H: C \rightarrow B$ so that for every $\delta \in \operatorname{Cov}(C)$ there is a proper $\delta$-small multi-valued function $L: X \rightarrow C$ so that $K \stackrel{\mu}{=} H \circ L$.

In order to check that $\tau$ is a cover we were looking for, let $Y$ be from the class $\mathcal{F}^{\prime \prime}$ and let $F: Y \rightarrow B$ be a proper $\tau$-small multi-valued function. Select a proper map $f: X \rightarrow Y$ from the class $\mathcal{F}$. Let $K$ denote the composition of $f$ and $F$. Notice that $K$ is a proper $\tau$-small multi-valued function from $X$ into $B$. Pick a space $C$ from the class $\mathcal{C}$ and a proper $\mu$-small multi-valued function $H$ as above. Let a $\gamma \in \operatorname{Cov}(C)$ be given. Let $\theta \in S(H, \mu)$ refines $\gamma$. Since $f$ is a proper $\mathcal{C} N$-surjection, there is a $\delta \in \theta^{+}$such that for every proper $\delta$-small multivalued function $L: X \rightarrow C$ there is a proper $\theta$-small multi-valued function $G: Y \rightarrow C$ with $L \stackrel{\theta}{\bumpeq} G \circ f$. Pick an $L$ and a $G$ as above. Our choices imply $F \circ f=K \stackrel{\mu}{\bumpeq} H \circ L \stackrel{\mu}{=} H \circ G \circ f$. It follows that $F \circ f \stackrel{\varrho}{=} H \circ G \circ f$ and therefore $F \stackrel{\sigma}{=} H \circ G$.

The situation with properly $P_{\mathcal{C}}^{\mathcal{B}}$-tame classes of spaces is much simpler as the following theorem shows. The proof of it is left to the reader.

THEOREM 3.17. If $\mathcal{F}$ is a class of proper $M^{\mathcal{c}}$-surjections and the class $\mathcal{F}^{\prime \prime}$ is properly $P_{\mathcal{C}}^{\mathcal{B}}$-tame, then the class $\mathcal{F}^{\prime}$ is also properly $P_{\mathcal{C}}^{\mathcal{B}}$-tame.

\section{PROPERLY $N^{\mathcal{B}}$-MOVABLE CLASSES.}

In this section we shall do for properly $M^{c}$-movable spaces what we have done in $\S 3$ for properly $M_{\mathcal{C}}^{\mathcal{B}}$-tame spaces. In other words, we shall introduce a dual notion called proper 
$N^{\mathcal{B}}$-movalility. It applies to classes of spaces and it satisfies six theorems which are analogues of results in $[5,(55]$.

Let $x^{\prime}$ and $\mathcal{B}$ be a classes of spares. The class $x$ is called properly $N^{\mathcal{B}}$-movable provided the class $\mathcal{B}$ is properly $M^{x^{\prime}}$-movalule, i. (e., provided for every $B \in \mathcal{B}$ and every $\sigma \in \operatorname{Cou}(B)$ there is a $\tau \in \operatorname{Cov}(B)$ such that for every $X \in \lambda$, every $\varrho \in \operatorname{Cov}(B)$, and every proper $\tau$-small multivalued function $F: X \rightarrow B$ these is a proper $\varrho$-small multi-valued function $G: X \rightarrow B$ with $F \stackrel{\sigma}{\bumpeq} G$. A space $X$ is properly $N^{\mathcal{B}}$-movable provided the class $\{X\}$ is properly $N^{\mathcal{B}}$-movable.

The following two theorems are immediate consequences of 5.2 and 5.1 in [5], respectively.

THEOREM 4.1. If a class of spaces $\mathcal{C}$ quasi $\mathcal{S} h_{p}$-dominates another such class $\mathcal{B}$ and a class of spaces $x^{\prime}$ is properly $N^{\mathcal{C}}$-movable, then the class $x^{\prime}$ is also properly $N^{\mathcal{B}}$-movable.

THEOREM 4.2. A class of spaces $\mathcal{x}$ will be properly $N^{\mathcal{B}}$-movable if and only if it is properly $M$-dominated by a properly $N^{\mathcal{B}}$-movable class of spaces $\mathcal{Y}$.

THEOREM 4.3. Let $\mathcal{B}$ and $\mathcal{C}$ be classes of topological spaces. If a class of spaces $X$ is at the same time properly $N_{\mathcal{C}}^{\mathcal{B}}$-tame and properly $N^{\mathfrak{C}}$-movable, then it is also properly $N^{\mathcal{B}}$-movable.

PROOF. Let a member $B$ of the class $\mathcal{B}$ and a numerable cover $\sigma$ of $B$ be given. Let $\pi \in \sigma^{*}$. Since $\mathcal{X}^{\prime}$ is properly $N^{c^{*}}$-movable, there is a numerable cover $\xi \in \pi^{+}$such that for every proper $\xi$-small multi-valued function $\Lambda$ from a member $C$ of $\mathcal{C}$ into $B$ and every $\varrho \in \operatorname{Cov}(X)$ there is a proper $\varrho$-small multi-valued function $L: C \rightarrow X$ with $K \stackrel{\pi}{=} L$. Since $\mathcal{X}$ is properly $N_{\mathcal{C}}^{\mathcal{B}}$-tame, there is a numerable cover $\tau$ of $B$ such that for every proper $\tau$-small multi-valued function $F$ of a member $X$ of $x$ into $B$ we can find a $C \in \mathcal{C}$ and a proper $\xi$-small multi-valued function $R: C \rightarrow B$ with the property that for every $\alpha \in \operatorname{Cov}(C)$ there is a proper $\alpha$-small multi-valued function $P: X \rightarrow C$ so that $F \stackrel{\xi}{\bumpeq} R \circ P$. Then $\tau$ is the required numerable cover of $B$.

Indeed, let $F$ be a proper $\tau$-small multi-valued function from a member $X$ of $\mathcal{X}$ into $B$ and let $\varrho$ be a numerable cover of $B$. Choose a $C$ and an $R$ as above. Then we pick a proper $\varrho$-small multi-valued function $K$ and a proper $\pi$-homotopy $L: C \times I \rightarrow B$ joining $R$ and $K$. Let $\alpha$ be a numerable cover of $C$ that satisfies $\alpha \in D(L, \pi)$ and $\alpha \in S(K, \varrho)$. With respect to $\alpha$ we now select a $P$ as above and put $G=K \circ P$. Then $G$ is a proper $\varrho$-small multi-valued function and we have $F \stackrel{\xi}{\bumpeq} R \circ P \stackrel{\pi}{\bumpeq} K \circ P=G$. Hence, $F \stackrel{\sigma}{\bumpeq} G$.

THEOREM 4.4. If a class of maps $\mathcal{F}$ is properly $N^{\mathcal{B}}$-surjective and the class $\mathcal{F}^{\prime \prime}$ is properly $N^{\mathcal{B}}$-movable, then the class $\mathcal{F}^{\prime}$ is also properly $N^{\mathcal{B}}$-movable.

PROOF. Let a member $B$ of $\mathcal{B}$ and a numerable cover $\sigma$ of $B$ be given. Let $\mu \in \sigma^{*}$. Since the class $\mathcal{F}^{\prime \prime}$ is properly $N^{\mathcal{B}}$-movable, there is a numerable cover $\xi \in \mu^{+}$of $B$ such that for every proper $\xi$-small multi-valued function $K: Y \rightarrow B$ from a member $Y$ of $\mathcal{F}^{\prime \prime}$ into $B$ and every $\varrho \in \operatorname{Cov}(B)$ there is a proper $\varrho$-small multi-valued function $L: Y \rightarrow B$ with $K \stackrel{\mu}{=} L$. On the other hand, since $\mathcal{F}$ is properly $N^{\mathcal{B}}$-surjective, there is a $\tau \in \operatorname{Cov}(B)$ such that for every proper map $f: X \rightarrow Y$ from the class $\mathcal{F}$ and every proper $\tau$-small multi-valued function $F: X \rightarrow B$ there is a proper $\xi$-small multi-valued function $K: Y \rightarrow B$ with $F \stackrel{\xi}{=} K \circ f$. Then $\tau$ is the numerable cover of $B$ we were looking for.

In fact, consider a proper $\tau$-small multi-valued function $F: X \rightarrow B$ from a member $X$ of $\mathcal{F}^{\prime}$ into $B$ and a $\varrho \in \operatorname{Cov}(B)$. Pick a proper map $f: X \rightarrow Y$ in $\mathcal{F}$ and proper functions $K$ and $L$ as above. Let $G$ denote the composition of $f$ and $L$. Then $G$ is a proper $\varrho$-small multi-valued function and we have $G=L \circ f \stackrel{\mu}{=} K \circ f \stackrel{\xi}{=} F$. Hence, $F \stackrel{\sigma}{\bumpeq} G$.

COROLLARY 4.5. The image of a properly $N^{\mathcal{B}}$-movable space by a refinable proper map is properly $N^{\mathcal{B}}$-movable space.

THEOREM 4.6. If $\mathcal{F}$ is a class of properly right $M$-placid proper maps and the class $\mathcal{F}^{\prime}$ is properly $N^{\mathcal{B}}$-movable, then the class $\mathcal{F}^{\prime \prime}$ is also properly $N^{\mathcal{B}}$-movalle.

PROOF. Let a member $B$ of $\mathcal{B}$ and a $\sigma \in \operatorname{Cov}(B)$ be given. Let $\mu \in \sigma^{*}$. Since the class $\mathcal{F}^{\prime}$ is properly $N^{\mathcal{B}}$-movable, there is a numerable cover $\tau \in \mu^{+}$with the property that for every proper $\tau$-small multi-valued function $K$ from a member $X$ of $\mathcal{F}^{\prime}$ into $B$ and every $\varrho \in \operatorname{Cov}(B)$ there is a proper $\varrho$-small multi-valued function $L: X \rightarrow B$ with $K_{\stackrel{\mu}{\wedge}}^{\wedge} L$. Then $\tau$ is the required numerable cover. 
In fact. let a member $I^{*}$ of $\mathcal{F}^{\prime \prime}$, a proper $\tau$-small multi-valued function $F: Y \rightarrow B$, and a numerable cover $\varrho$ of $B$ be given. Let $f: X \rightarrow Y^{-}$be $f_{1}$ om the class $\mathcal{F}$. We let $K$ denote the composition $F \circ f$ and pick an $L$ as above. Let $W$ be a proper $\mu$-homotopy joining $K$ and $L$. Let $\theta \in \operatorname{Cov}(X)$ be from the intersection of sets $S(L, \varrho)$ and $D(W, \mu)$. Let $\alpha \in S(F, \tau)$. Since the map $f$ is properly right $M$-placid, there is a proper $\theta$-small multi-valued function $J: Y \rightarrow X$ such that $i d_{Y} \stackrel{\propto}{\simeq} f \circ J$. Let $G$ be the composition of $J$ and $L$. The function $G$ is proper and $\varrho$-small and we have relations $G=L \circ J \stackrel{\mu}{\bumpeq} K \circ J=F \circ f \circ J \stackrel{r}{=} F$. Hence, $F \stackrel{\sigma}{\bumpeq} G$.

THEOREM 4.7. If $\mathcal{F}$ is a properly $N^{\mathcal{B}}$-injective class of proper $N^{\mathcal{B}}$-surjections and $\mathcal{F}^{\prime}$ is properly $N^{\mathcal{B}}$-movalble, then $\mathcal{F}^{\prime \prime}$ is also properly $N^{\mathcal{B}}$-movable.

PROOF. Let a member $B$ of $\mathcal{B}$ and a numerable cover $\sigma$ of $B$ be given. Since the class $\mathcal{F}$ is properly $N^{\mathcal{B}}$-injective, there is a $\mu \in \operatorname{Cor}(B)$ such that for every proper map $f: X \rightarrow Y$ from $\mathcal{F}$ and all proper $\mu$-small multi-valued functions $F, G: Y \rightarrow B$ the relation $F \circ f \stackrel{\xi}{=} G \circ f$ implies the relation $F \stackrel{\sigma}{\bumpeq} G$. Let $\lambda \in \mu^{*}$. Since the class $\mathcal{F}^{\prime}$ is properly $N^{\mathcal{B}}$-movable, there is a $\tau \in \operatorname{Cov}(B)$ such that for every member $X$ of $\mathcal{F}^{\prime}$, every proper $\tau$-small multi-valued function $P: X \rightarrow B$ and every $\pi \in \operatorname{Cov}(B)$ there is a proper $\pi$-small multi-valued function $Q: X \rightarrow B$ with $P \stackrel{\lambda}{=} Q$. Then $\tau$ is the required numerable cover of $B$.

In order to verify this, consider a member $Y$ of $\mathcal{F}^{\prime \prime}$, a proper $\tau$-small multi-valued function $F: Y \rightarrow B$, and a numerable cover $\varrho \in \operatorname{Cov}(B)$. We can assume that $\varrho$ refines $\lambda$. Let $f: X \rightarrow Y$ be from $\mathcal{F}$. Since $f$ is a proper $N^{\mathcal{B}}$-surjection, there is a $\pi \in \operatorname{Cov}(B)$ such that for every proper $\pi$-small multi-valued function $Q: X \rightarrow B$ there is a proper $\varrho$-small multivalued function $G: Y \rightarrow B$ with $Q \stackrel{\delta}{=} G \circ f$. Let $P$ denote the composition of $f$ and $F$. Observe that $P$ is a proper $\tau$-small multi-valued function from $X$ into $B$. Pick a $Q$ and a $G$ as above. Our selections imply the following relations : $F \circ f=P \stackrel{\lambda}{=} Q \stackrel{\ell}{=} G \circ f$. It follows that $F \circ f \stackrel{\xi}{=} G \circ f$ and therefore $F \stackrel{\sigma}{\bumpeq} G$.

\section{COVERED AND EXTENDED CLASSES.}

In this section we shall explore dependence of all proper shape invariants which were defined on classes of spaces involved under the assumption that these classes are connected by either surjections or injections. The connection can be through one of the following two notions.

Let $\mathcal{F}$ be a class of proper maps and let $\mathcal{B}$ and $\mathcal{C}$ be classes of spaces. We shall say that the class $\mathcal{C}$ is $\mathcal{F}$-covered by $\mathcal{B}$ provided for every $C \in \mathcal{C}$ there is a $B \in \mathcal{B}$ and an $h: B \rightarrow C$ from $\mathcal{F}$. Similarly, the class $\mathcal{C}$ is $\mathcal{F}$-extended by $\mathcal{B}$ provided for every $C \in \mathcal{C}$ there is a $B \in \mathcal{B}$ and a $k: C \rightarrow B$ from $\mathcal{F}$.

For a class of spaces $\mathcal{B}$ we shall use $\mathcal{B}_{z}, \mathcal{B}_{s}$, and $\mathcal{B}_{b}$ to denote the classes of all proper $M^{\mathcal{B}}$-injections, proper $M^{\mathcal{B}}$-surjections, and proper $M^{\mathcal{B}}$-bijections. Also, $\mathcal{B}^{z}, \mathcal{B}^{s}$, and $\mathcal{B}^{b}$ denote the classes of all proper $N^{\mathcal{B}}$-injections, proper $N^{\mathcal{B}}$-surjections, and proper $N^{\mathcal{B}}$-bijections. Moreover, if $\mathcal{F}$ and $\mathcal{G}$ are classes of maps we let $\mathcal{F G}$ denote the intersection $\mathcal{F} \cap \mathcal{G}$.

We begin with the result on properly $N_{\mathcal{C}}^{\mathcal{B}}$-tame spaces and continue to cover all our proper shape invariants. The proofs are mostly omitted.

THEOREM 5.1. Let $\mathcal{A}, \mathcal{B}, \mathcal{C}, \mathcal{D}$, and $\mathcal{X}$ be classes of spaces such that $\mathcal{X}$ is properly $N_{\mathcal{D}}^{\mathcal{A}}$-tame and either

(cc) $\mathcal{B}$ is $\mathcal{X}_{s}$-covered by $\mathcal{A}$ and $\mathcal{D}$ is $\mathcal{X}_{s}$-covered by $\mathcal{C}$,

(ce) $\mathcal{B}$ is $\mathcal{X}_{s}$-covered by $\mathcal{A}$ and $\mathcal{D}$ is $\mathcal{A}^{s}$-extended by $\mathcal{C}$,

(ec) $\mathcal{B}$ is $\mathcal{C}_{s} \mathcal{X}_{i}$-extended by $\mathcal{A}$ and $\mathcal{D}$ is $\mathcal{X}_{s}$-covered by $\mathcal{C}$, or

(ee) $\mathcal{B}$ is $\mathcal{C}_{s} \mathcal{X}_{2}$-extended by $\mathcal{A}$ and $\mathcal{D}$ is $\mathcal{A}^{s}$-extended by $\mathcal{C}$,

then $\mathcal{X}$ is also properly $N_{\mathcal{C}}^{\mathcal{B}}$-tame.

PROOF. (cc). Let a member $B$ of $\mathcal{B}$ and a numerable cover $\sigma$ of $B$ be given. Let $\mu \in \sigma^{*}$. Since the class $\mathcal{B}$ is $\mathcal{X}_{s}$-covered by the class $\mathcal{A}$, there is an $A \in \mathcal{A}$ and a proper $M^{\mathcal{X}}$-surjection $h: A \rightarrow B$. Let $\delta=h^{-1}(\mu)$. We now utilize the assumption that the class $\mathcal{X}$ is properly $N_{\mathcal{D}}^{\mathcal{A}}$-tame to select a numerable cover $\varepsilon$ with the property that for every proper $\varepsilon$-small multivalued function $P$ from a member $X$ of $x$ into $A$ there is a $D \in \mathcal{D}$ and a proper $\delta$-small 
multi-valued function $I: D \rightarrow A$ so that for every $\eta \in \operatorname{Cor}(D)$ there is a proper $\eta$-small multi-valued function $L: X \rightarrow D$ with $P \stackrel{\wp}{\bumpeq} K \circ L$. Since $h$ is a proper $M^{x}$-surjection, there is a $\tau \in \operatorname{Cov}(B)$ such that for every $X \in \lambda$ and every proper $\tau$-small multi-valued function $F: X \rightarrow B$ there is a proper $\varepsilon$-small multi-valued function $P: X \rightarrow A$ with $F \stackrel{\mu}{\bumpeq} h \circ P$. Then $\tau$ is a numerable cover we were looking for.

Indeed, let $X \in x$ and let $F: X \rightarrow B$ be a proper $\tau$-small multi-ralued function. Pick a $P$ and then a $D$ and a $K$ as above. Since the class $\mathcal{D}$ is $\mathcal{X}_{4}$-covered by the class $\mathcal{C}$, there is a

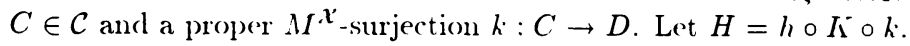

Let $\gamma$ be a numerable cover of $C$. Let $\theta \in S(K, \delta)$. We nse now the fact that $k$ is a proper $M^{x^{\prime}}$-surjection to choose an $\eta \in \operatorname{Cov}(D)$ with the property that for every $X \in \mathcal{X}^{\prime}$ and every proper $\eta$-small multi-valued function $K^{-}: X \rightarrow D$ there is a proper $\gamma$-small multi-valued function $G: X \rightarrow C$ with $L \stackrel{\theta}{=} k \circ G$. Pick an $L$ and then a $G$ as above. Our selections imply the following chain of relations: $F \stackrel{\mu}{\bumpeq} h \circ P \stackrel{\mu}{\bumpeq} h \circ K \circ L \stackrel{\mu}{\bumpeq} h \circ K \circ k \circ G=H \circ G$. Hence, $F \stackrel{\sigma}{=} H \circ G$.

THEOREM 5.2. Let $\mathcal{A}, \mathcal{B}, \mathcal{C}, \mathcal{D}$, and $\mathcal{X}$ be classes of spaces such that $\mathcal{X}$ is properly $P_{\mathcal{D}}^{\mathcal{A}}$-tame and either

(cc) $\mathcal{B}$ is $\mathcal{D}_{s}$-covered by $\mathcal{A}$ and $\mathcal{C}$ is $\mathcal{B}_{2}, \mathcal{X}^{\prime s}$-covered by $\mathcal{D}$,

(ce) $\mathcal{B}$ is $\mathcal{D}_{s}$-covered by $\mathcal{A}$ and $\mathcal{C}$ is $\mathcal{B}^{s}$-extended by $\mathcal{D}$,

(ec) $\mathcal{B}$ is $\mathcal{D}_{2} \mathcal{X}_{s}$-extended by $\mathcal{A}$ and $\mathcal{D}$ is $\mathcal{B}^{2} \mathcal{X}^{\prime}$-covered by $\mathcal{D}$, or

(ee) $\mathcal{B}$ is $\mathcal{C}_{1} \mathcal{D}_{s}$-extended by $\mathcal{A}$ and $\mathcal{C}$ is $\mathcal{B}^{s}$-extended by $\mathcal{D}$,

then $\mathcal{X}$ is also properly $P_{\mathcal{C}}^{\mathcal{B}}$-tame.

THEOREM 5.3. Let $\mathcal{X}, \mathcal{B}$, and $\mathcal{C}$ be classes of spaces. If the class $\mathfrak{X}^{\prime}$ is properly $N^{\mathcal{B}}$ movable and the class $\mathcal{C}$ is either $\mathfrak{x}_{s}$-covered or $\mathcal{X}_{b}^{\prime}$-extended by $\mathcal{B}$, then $\mathcal{x}$ is also properly $N^{\mathcal{C}}$-movable.

PROOF. ( $\mathcal{C}$ is $\boldsymbol{x}_{s}$-covered by $\left.\mathcal{B}\right)$. Let a member $C$ of $\mathcal{C}$ and a numerable cover $\gamma$ of $C$ be given. Let $\theta \in \gamma^{*}$. Since the class $\mathcal{C}$ is $\mathcal{X}_{s}^{\prime}$-covered by the class $\mathcal{B}$, there is a $B \in \mathcal{B}$ and a

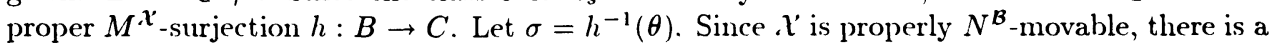
numerable cover $\tau \in \operatorname{Cov}(B)$ such that for every proper $\tau$-small multi-valued function $K$ from a member of $X$ into $B$ and every $\varrho \in \operatorname{Cov}(B)$ there is a proper $\varrho$-small multi-valued function

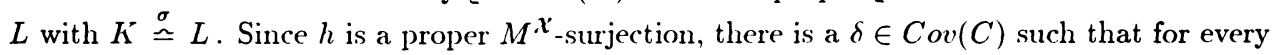
proper $\delta$-small multi-valued function $F$ from a member of $\mathcal{X}$ into $C$ there is a proper $\tau$-small multi-valued function $K$ with $F \stackrel{\theta}{=} h \circ K$. Then $\delta$ is the required numerable cover of $C$.

In fact, let $\varepsilon$ be an arbitrary numerable cover of $C$, let $X \in \mathcal{X}$, and let $F: X \rightarrow C$ be a proper $\delta$-small multi-valued function. Let $\varrho=h^{-1}(\varepsilon)$. Pick a $K$ and an $L$ as above and let $G$ denote the composition $h \circ L$.

Then $G$ is a proper $\varepsilon$-small multi-valued function and the relation $K \stackrel{\sigma}{\bumpeq} L$ implies $F \stackrel{\theta}{\bumpeq}$ $h \circ K \stackrel{\theta}{=} h \circ L=G$. Hence, $F \stackrel{\gamma}{=} G$.

\section{REFERENCES}

1. BALL, B. J. and SHER, R. B., A theory of proper shape for locally compact spaces, Fund. Math. 86, (1974), pp. 163 - 192.

2. BORSUK, K., Theory of Shape, PNW, Warszawa, (1975).

3. ČERIN, Z., Proper shape theory, Acta Scientiarum Mathematicarum, (1994), to appear.

4. ČERIN, Z., Morphisms in proper shape theory, Math. Japonica, (1994), to appear.

5. ČERIN, Z. and G.M. GIANELLA, Tameness and movability in proper shape theory, preprint.

6. DOLD, A., Lectures on Algebraic Topology, Springer-Verlag, Berlin, (1972).

7. SIEBENMANN, L. C., The obstruction to finding a boundary for an open manifold of dimension greater than five, Thesis, Princeton University, (1965). 


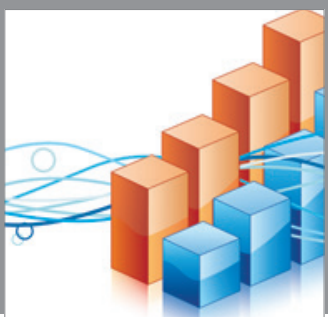

Advances in

Operations Research

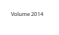

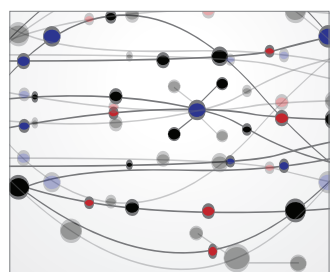

\section{The Scientific} World Journal
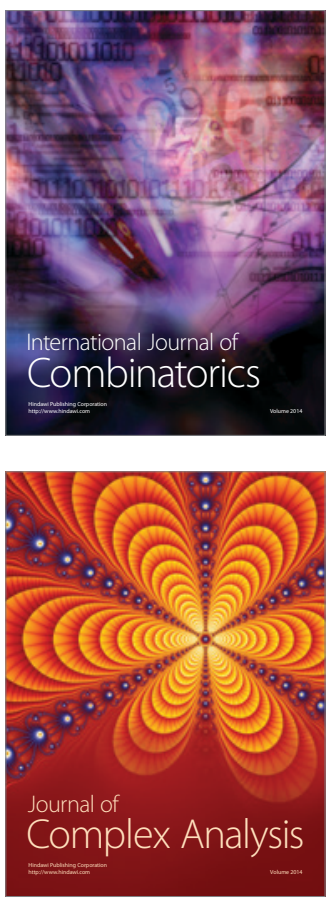

International Journal of

Mathematics and

Mathematical

Sciences
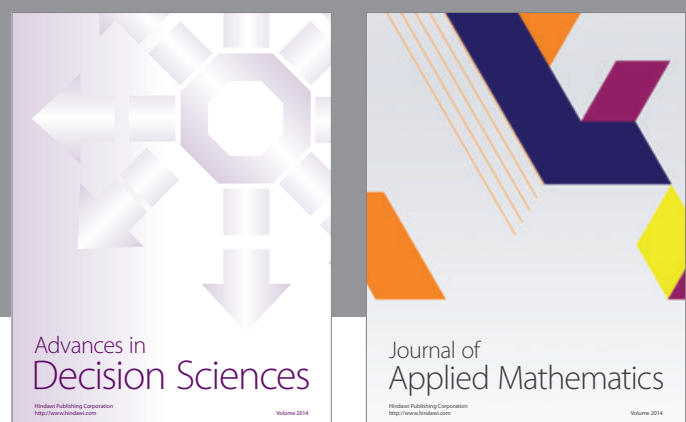

Journal of

Applied Mathematics
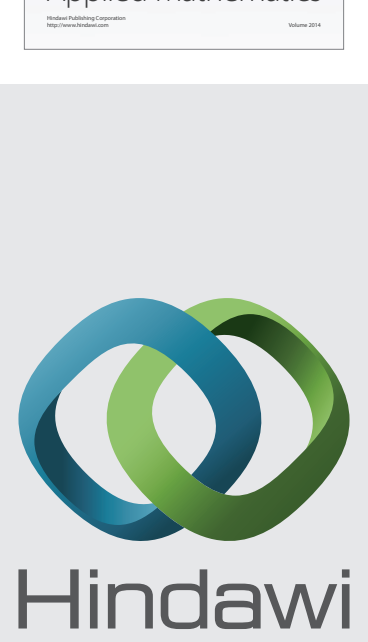

Submit your manuscripts at http://www.hindawi.com
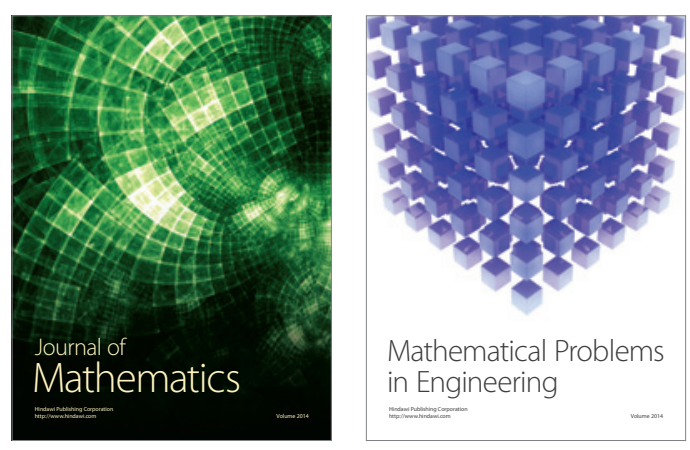

Mathematical Problems in Engineering
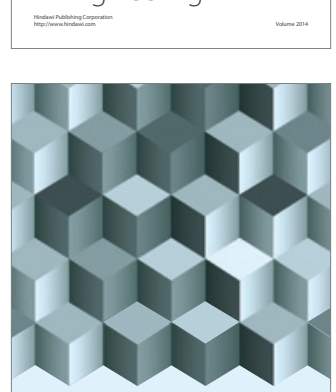

Journal of

Function Spaces
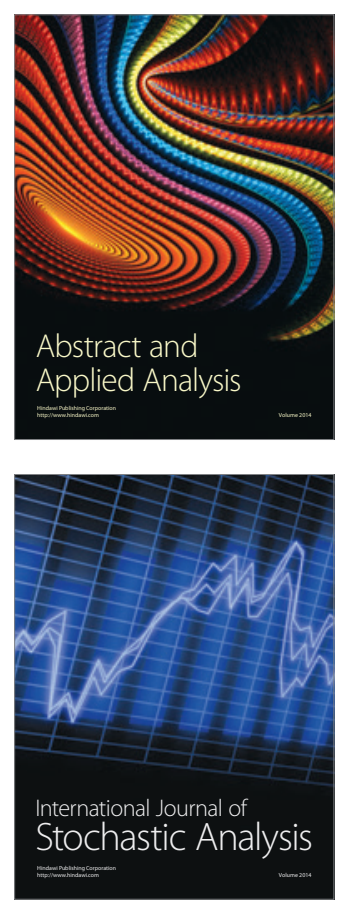

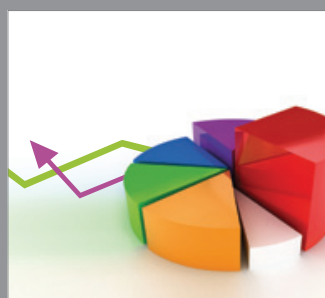

ournal of

Probability and Statistics

Promensencen
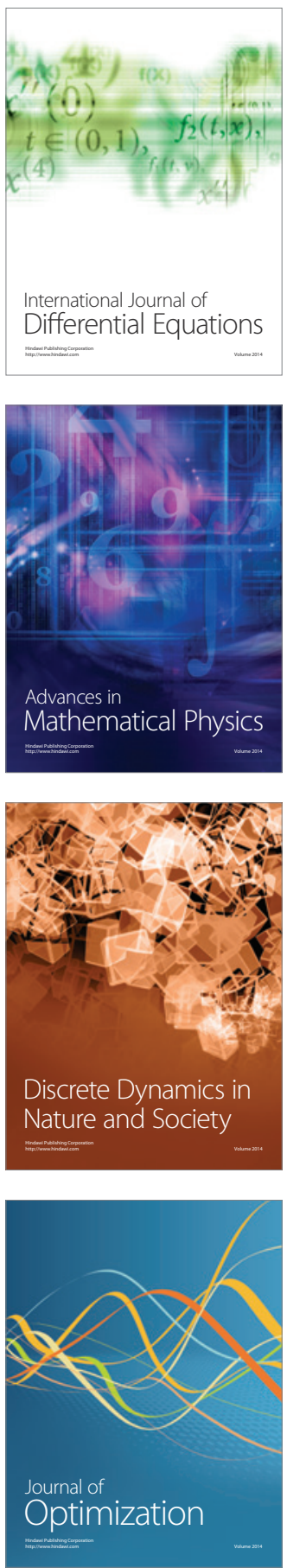